\title{
Fluoroquinolone exposure prior to tuberculosis diagnosis is associated with an increased risk of death
}

\author{
Y. F. van der Heijden ${ }^{*}$, F. Maruri ${ }^{*}$, A. Blackman ${ }^{*}$, E. Holt ${ }^{\star}, \dagger$, J. V. Warkentin ${ }^{\dagger}$, B. E. \\ Shepherd ${ }^{\ddagger}$, and T. R. Sterling ${ }^{\star}, \S$ \\ *Division of Infectious Diseases, Department of Medicine, Vanderbilt University School of \\ Medicine, Nashville, Tennessee \\ †Tennessee Department of Health, Nashville, Tennessee \\ ‡Department of Biostatistics, Vanderbilt University School of Medicine, Nashville, Tennessee, \\ USA
}

$\S$ Center for Health Services Research, Department of Medicine, Vanderbilt University School of Medicine, Nashville, Tennessee, USA

\section{SUMMARY}

SETTING—Fluoroquinolone (FQ) exposure before tuberculosis (TB) diagnosis is common, but its effect on outcomes, including mortality, is unclear.

DESIGN_Among TB patients reported to the Tennessee Department of Health from 2007 to 2009, we assessed FQ exposure within 6 months before TB diagnosis. The primary outcome was the combined endpoint of death at the time of TB diagnosis and during anti-tuberculosis treatment.

RESULTS-Among 609 TB cases, 214 (35\%) received FQs within 6 months before TB diagnosis. A total of 71 (12\%) persons died; 10 (2\%) were dead at TB diagnosis and 61 (10\%) died during anti-tuberculosis treatment. In multivariable logistic regression analysis, factors independently associated with death were older age (OR 1.05 per year, 95\% CI 1.04-1.07), human immunodeficiency virus infection (OR 8.08, 95\% CI 3.83-17.06), US birth (OR 3.03, 95\%CI 1.03-9.09), and any FQ exposure before TB diagnosis (OR 1.82, 95\% CI 1.05-3.15). Persons with FQ exposure before TB diagnosis were more likely to have culture- and smear-positive disease than unexposed persons.

CONCLUSIONS-Among this patient population, FQ exposure before TB diagnosis was associated with an increased risk of death. These findings underscore the need for cautious use of FQs in persons with possible TB.

\section{Keywords}

drug-susceptible TB; drug resistance; mortality

Despite a Decrease in worldwide incidence since 2002, tuberculosis (TB) continues to be a major cause of mortality worldwide; it is estimated that 1.45 million persons died from TB in 2010. ${ }^{1}$ Factors that increase the risk of TB-associated death include older age, human

\footnotetext{
(C) 2012 The Union; 1-6

Correspondence to: Yuri F van der Heijden, Vanderbilt University Medical Center, A2200 Medical Center North, 116121 st Avenue South, Nashville, TN 37232, USA. Tel: (+1) 615322 8972. Fax: (+1) 615343 6160. yuri.vanderheijden@ vanderbilt.edu.

Data from this study were presented at the American Thoracic Society International Conference, Denver, Colorado, USA, 15 May 2011 (Abstract \# 16983).
} 
immunodeficiency virus (HIV) infection, being Black, Hispanic and homeless, having miliary or meningeal TB, malignancy, frequent alcohol use and drug-resistant disease. ${ }^{2-6}$ Multidrug-resistant TB (MDR-TB), defined as resistance to at least isoniazid (INH) and rifampin (RMP), and extensively drug-resistant TB (XDR-TB), defined as MDR-TB strains that are also resistant to fluoroquinolones (FQs) and a second-line injectable agent, are of growing global concern.

FQs demonstrate excellent in vitro and in vivo activity against Mycobacterium tuberculosis,, 78 and are important components of the treatment regimens for MDR-TB and in patients who are intolerant of first-line treatment. Because of their broad antimicrobial properties, outstanding bioavailability and relatively low toxicity, FQs are commonly used to treat a variety of bacterial infections. The prevalence of FQ exposure in the 12 months prior to TB diagnosis has been as high as $41 \% .^{9}$

The use of FQs prior to TB diagnosis has been associated with an increased risk of FQ resistance ${ }^{10-12}$ and delays in diagnosis and treatment of pulmonary TB. ${ }^{13-17}$ One retrospective study in a TB-endemic area found that FQ exposure before TB diagnosis was associated with increased mortality. ${ }^{14}$ We sought to clarify the association between FQ exposure before TB diagnosis and mortality in an area of low TB incidence using a prospective cohort with both in- and out-patient FQ exposure data.

\section{METHODS}

We performed a prospective cohort study among patients with newly diagnosed TB reported to the Tennessee Department of Health (DoH) from January 2007 to December 2009. Patients from the cohort were included if they had an assessment for possible FQ exposure starting 6 months before TB diagnosis from at least one of four sources. An FQ exposure assessment survey was completed by Tennessee DoH staff who questioned TB patients regarding receipt of FQs. An in-home questionnaire recorded data from face-to-face interviews of patients with culture-positive TB conducted by research study staff regarding patient recall of FQ prescriptions. Research study staff reviewed clinic and hospital records to assess details of FQ prescriptions among culture-positive patients who gave consent and for any culture-positive patients who were dead at diagnosis, died during treatment or died after completion of anti-tuberculosis treatment. For each of the four sources of FQ exposure data, the names of specific FQs prescribed during the 6 months preceding TB diagnosis, date of prescription, duration of prescription and reason for prescription were ascertained where possible.

The study was approved by the institutional review boards of Vanderbilt University, the Tennessee DoH, and the Davidson County Metro Public Health Department.

The primary outcome of the study was all-cause mortality as a combined endpoint of death at the time of TB diagnosis and death during anti-tuberculosis treatment. Secondary outcomes included death at either time point alone, site of disease, pulmonary cavitation and acid-fast smear and culture status. The date of TB diagnosis was defined as the date of initiation of anti-tuberculosis treatment. The date of death was obtained from the Tennessee DoH database, local health departments, the Tennessee DoH Division of Health Statistics or the Social Security Death Index Database. Deaths were ascertained through October 2010.

Culture-positive TB was defined as cases in which Mycobacterium tuberculosis was isolated at least once from sputum, tissue or body fluid cultures. Cases were defined as culturenegative TB when no cultures grew M. tuberculosis and any of the following occurred: 1) nucleic acid amplification testing of a clinical specimen demonstrated M. tuberculosis; 2) 
acid-fast bacilli were confirmed on a sputum smear or a smear, pathology or cytology specimen of other body fluids or tissue; 3 ) the case met clinical case definition criteria (positive tuberculin skin test or positive interferon gamma release assay for M. tuberculosis, abnormal plain chest radiograph [CXR] or chest computed tomography scan if the site of disease was pulmonary, pleural, or intrathoracic lymphatic, and treatment with at least two anti-tuberculosis medications); or 4) the case met physician-verified TB criteria (culturenegative cases who did not meet the clinical case definition but were considered cases by physicians at the Tennessee DoH). Patients with culture-negative TB had to demonstrate clinical response to treatment.

Patients with pulmonary TB had M. tuberculosis isolated from or evidence of disease in sputum, bronchoalveolar lavage or lung parenchyma. If specimens from anywhere outside of the lung parenchyma were positive for M. tuberculosis, patients were considered to have extra-pulmonary TB. Patients with evidence of cavitation on plain CXR were considered to have cavitary disease.

Clinical specimens were sent to the Tennessee DoH Division of Laboratory Services Special Microbiology Section for fluorochrome acid-fast stain and culture, either directly, or for further testing if they were positive, at outside laboratories. Identification of mycobacteria was performed by high performance liquid chromatography or nucleic acid hybridization (AccuProbe, Gen-Probe Inc, San Diego, CA, USA).

FQ exposure was characterized as a categorical variable (exposed vs. unexposed). Duration of FQ exposure was characterized categorically (>10 days vs. 1-10 days) and as a continuous variable (cumulative days of exposure). Differences in demographic and clinical characteristics between FQ-exposed and -unexposed groups were evaluated using the $\chi^{2}$ test for categorical variables and the Wilcoxon rank-sum test for continuous variables. Factors associated with death were assessed using univariate and multivariable logistic regression analysis, and included age, sex, race, HIV status, birth in the United States, MDR-TB, resistance to any first-line anti-tuberculosis medications and FQ exposure. Patients who were dead at diagnosis were not included in the logistic regression analysis of factors associated with death during treatment. A Cox proportional hazards model was used to evaluate differences in time to death in the first 6 months (specifically 182 days) after starting treatment between those who received FQs before TB diagnosis and those who did not. For cases in which HIV status was missing, multiple imputation techniques were used to predict HIV status in regression and hazards models.

All analyses were performed using Stata, version 10 (Stata Corporation, College Station, TX, USA), except for multiple imputation and Cox proportional hazards calculations, which were performed using $R$ (R statistical software, version 2.11.1, Vienna, Austria, www.rproject.org). All $P$ values were two-sided and $P \leq 0.05$ was considered significant.

\section{RESULTS}

Of the 719 TB cases reported to the Tennessee DoH during the study period, 609 were included in the study as data were available on possible FQ exposure from at least one source. The 110 excluded patients were significantly younger and were less likely to be USborn than the included cases. Of the 609 cases, 390 (64\%) were male, 238 (39\%) were Black and 434 (71\%) were US-born; 586 had HIV testing results available, of whom 59 (10\%) were HIV-positive.

The majority of the patients ( $n=500,82 \%)$ had pulmonary TB, including $36(6 \%)$ who had both pulmonary and extra-pulmonary disease. CXR revealed cavitary disease in $150(25 \%)$ 
patients. Smear-positive TB occurred in $241(40 \%)$ patients and $460(76 \%)$ were culturepositive. Of $454 \mathrm{M}$. tuberculosis isolates tested, $13(3 \%)$ were resistant to INH and 3/455 (1\%) were resistant to RMP; all three of the RMP-resistant isolates were also INH-resistant (i.e., MDR-TB). FQ susceptibility testing was performed on 441 (72\%) isolates, nine (2\%) of which were FQ-resistant.

Among the study patients, 214 (35\%) received at least one FQ prescription within the 6 months before TB diagnosis. Demographic and clinical characteristics of the study patients categorized according to FQ exposure are listed in Table 1. Those who had any FQ exposure were significantly older and more likely to be US-born or Hispanic than those who were unexposed. Table 2 lists FQ exposure characteristics of the study population.

Among the 609 TB cases, $71(12 \%)$ died: $10(2 \%)$ were dead at the time of TB diagnosis and $61(10 \%)$ died during anti-tuberculosis treatment. As noted in Table 3, when all deaths were considered as a combined endpoint, exposure to any FQ was associated with death in univariate logistic regression analysis. In multivariable logistic regression analysis adjusting for age, HIV status and US birth, FQ exposure was independently associated with death (odds ratio $[\mathrm{OR}] 1.82,95 \%$ confidence interval $[\mathrm{CI}] 1.05-3.15, P=0.03$ ) in addition to age, being HIV-positive and US-born.

Among those treated for TB (i.e., all patients except the 10 who were dead at TB diagnosis), FQ exposure was significantly associated with death during anti-tuberculosis treatment in both univariate (OR 2.63, 95\% CI 1.54-4.50, $P<0.001)$ and multivariable (OR 1.79, 95\% CI 1.01-3.17, $P=0.05$ ) logistic regression analyses, along with age, HIV-positive status and US birth. Only age and HIV-positive status, however, were associated with death at the time of TB diagnosis in multivariable regression analysis; any $\mathrm{FQ}$ exposure was not significantly associated with death at the time of TB diagnosis in either univariate (OR 2.82, 95\%CI $0.79-10.10, P=0.11$ ) or multivariable (OR $1.51,95 \% \mathrm{CI} 0.37-6.24, P=0.57$ ) logistic regression models.

FQ exposure was independently associated with death when all deaths were considered and after imputation of HIV status for those in whom it was unknown, as reported in Table 3, but not when the 23 patients with unknown HIV status were excluded (OR 1.68, 95\% CI 0.93$3.02, P=0.08)$. Patients with unknown HIV status were significantly more likely to be older and US-born and less likely to be Black than those whose HIV status was known. Patients with unknown HIV status were also significantly more likely to have had FQ exposure, to have been dead at diagnosis or to have died during treatment.

We observed a difference in the rates of death in the first 6 months after initiation of treatment between those who were exposed to FQs in the 6 months before TB diagnosis and those who were not (Figure). The difference in rates of death between those exposed to FQs before TB diagnosis and those unexposed appeared to be most pronounced in the first 30-60 days, based on the Figure. Taking into account age, HIV status and US birth, we observed a significant difference in the risk of death in the first 6 months after starting anti-tuberculosis treatment between those exposed to FQs and those unexposed using a Cox proportional hazards model (relative risk 1.78, 95\% CI 1.05-3.02, $P=0.03$ ).

Among the clinical, radiological and bacteriological characteristics evaluated, FQ exposure was associated with culture-positive $(P<0.001)$ and smear-positive TB $(P<0.001)$. Site of disease and cavitary disease were not associated with FQ exposure (Table 4). 


\section{DISCUSSION}

Although previous studies have investigated risk factors for mortality among persons with $\mathrm{TB},{ }^{2-6,18,19}$ our study is the first to evaluate the risk of death associated with FQ exposure prior to diagnosis in a low TB incidence area (3.2 cases per 100000 population in 2009) ${ }^{20}$ using in- and out-patient exposure data in a prospective cohort. One previous study conducted at a Taiwanese tertiary care referral center also demonstrated an independent association between FQ exposure and mortality, but ascertainment of FQ exposure was limited to review of hospital and TB case manager records. That study was performed in a region with much higher TB incidence (62.4 cases/100 000 in 2003). ${ }^{14}$

Previous studies have demonstrated an association between FQ exposure and delays in diagnosis and treatment of TB, and several studies have compared exposure to FQs vs. nonFQ antibiotics. Two of these found that those who received FQs were not more likely than recipients of non-FQ antibiotics to have delays in the initiation of anti-tuberculosis treatment or diagnosis of pulmonary TB. ${ }^{17,21}$ Two other studies, however, did report significant delays in treatment that were specifically attributable to FQs. A meta-analysis that looked at three of the four studies above and one additional study ${ }^{16}$ found a mean difference of 19.03 days (95\% CI 10.87-27.18, $P<0.001)$ in overall delays in starting anti-tuberculosis treatment between those who received FQs and those who received non-FQ antibiotics. ${ }^{22}$ Although TB treatment delays could arise from clinical decisions to monitor improvement after any empiric course of antibiotics, the above results suggest that FQs may have unique effects, such as their activity against $M$. tuberculosis, that additionally obscure the diagnosis of TB. While our study did not assess for delays in diagnosis and treatment of TB, these are conceivable mechanisms for the increased mortality associated with FQ exposure described here.

For survival analysis, we focused on the first 6 months after the initiation of antituberculosis treatment, as this represents the usual treatment duration for drug-susceptible TB. Although we did not have data on cause of death, it is possible that deaths occurring shortly after TB diagnosis were more likely to be due to TB than those that occurred later, particularly as the difference in rates of death between FQ-exposed and -unexposed groups appeared to be most pronounced within the first 60 days of TB diagnosis. Our cohort had relatively few persons who were dead at the time of TB diagnosis, which may have affected our ability to detect any association with prior FQ exposure.

It is interesting to note that persons with FQ exposure were more likely to have smear- and culture-positive TB. Although this finding is consistent with an earlier study that used data from the Tennessee DoH TB registry and TennCare (Medicaid) database from 2000-2004, ${ }^{9}$ the reasons are unclear.

Our study had several limitations. Prescriptions for FQs were used as a marker for FQ exposure, and we did not account for possible non-adherence. Due to the relatively small number of deaths in the cohort and patient data limitations, we were unable to adjust for comorbidities such as chronic obstructive pulmonary disease, diabetes mellitus or alcoholism. Furthermore, missing data could have affected the analyses. In particular, 23 (4\%) patients had unknown HIV status; when they were excluded from the analysis, the association between FQ exposure and mortality was not present. When we used multiple imputation to predict HIV status of those with unknown HIV status, however, FQ exposure was independently associated with death.

In this study, we demonstrated an association between FQ exposure prior to TB diagnosis and an increased risk of death. Our findings underscore the importance of keeping TB as a 
diagnostic consideration, even in areas of low TB incidence. In those patients who have risk factors for TB, an alternative to FQs should be considered if empiric antibiotic treatment is warranted. Further investigation in areas of high TB incidence and frequent use of FQs is needed, particularly given the growing concerns about drug-resistant TB.

\section{Acknowledgments}

The authors thank E Mitchel for his work involving FQ exposure data and C May for her work with drug resistance testing. This work was supported by the National Institute of Allergy and Infectious Diseases at the National Institutes of Health [grant numbers R01 AI063200, K24 AI65298, T32 AI07474-16]. TRS received research grant funding from Pfizer and Bristol Myers Squibb for HIV observational studies, and served on a Data Safety Monitoring Board for a study funded by Otsuka Pharmaceutical.

\section{References}

1. World Health Organization. WHO/HTM/TB/2011.16. Geneva, Switzerland: WHO; 2011. Global tuberculosis control: WHO report 2011. http://www.who.int/tb/publications/global_report/2011/ gtbr11_full.pdf [Accessed May 2012]

2. Pablos-Mendez A, Sterling TR, Frieden TR. The relationship between delayed or incomplete treatment and all-cause mortality in patients with tuberculosis. JAMA. 1996; 276:1223-1228. [PubMed: 8849749]

3. Marks SM, Magee E, Robison V. Patients diagnosed with tuberculosis at death or who died during therapy: association with the human immunodeficiency virus. Int J Tuberc Lung Dis. 2011; 15:465470. [PubMed: 21396204]

4. Nguyen LT, Hamilton CD, Xia Q, Stout JE. Mortality before or during treatment among tuberculosis patients in North Carolina, 1993-2003. Int J Tuberc Lung Dis. 2011; 15:257-262. [PubMed: 21219691]

5. Fielder JF, Chaulk CP, Dalvi M, Gachuhi R, Comstock GW, Sterling TR. A high tuberculosis casefatality rate in a setting of effective tuberculosis control: implications for acceptable treatment success rates. Int J Tuberc Lung Dis. 2002; 6:1114-1117. [PubMed: 12546121]

6. Sterling TR, Zhao Z, Khan A, et al. Mortality in a large tuberculosis treatment trial: modifiable and non-modifiable risk factors. Int J Tuberc Lung Dis. 2006; 10:542-549. [PubMed: 16704037]

7. Ginsburg AS, Grosset JH, Bishai WR. Fluoroquinolones, tuberculosis and resistance. Lancet Infect Dis. 2003; 3:432-442. [PubMed: 12837348]

8. Hu Y, Coates AR, Mitchison DA. Sterilizing activities of fluoroquinolones against rifampin-tolerant populations of Mycobacterium tuberculosis. Antimicrob Agents Chemother. 2003; 47:653-657. [PubMed: 12543673]

9. Gaba PD, Haley C, Griffin MR, et al. Increasing out-patient fluoroquinolone exposure before tuberculosis diagnosis and impact on culture-negative disease. Arch Intern Med. 2007; 167:23172322. [PubMed: 18039990]

10. Long R, Chong H, Hoeppner V, et al. Empirical treatment of community-acquired pneumonia and the development of fluoroquinolone-resistant tuberculosis. Clin Infect Dis. 2009; 48:1354-1360. [PubMed: 19348594]

11. Ginsburg AS, Hooper N, Parrish N, et al. Fluoroquinolone resistance in patients with newly diagnosed tuberculosis. Clin Infect Dis. 2003; 37:1448-1452. [PubMed: 14614666]

12. Devasia RA, Blackman A, Gebretsadik T, et al. Fluoroquinolone resistance in Mycobacterium tuberculosis: the effect of duration and timing of fluoroquinolone exposure. Am J Respir Crit Care Med. 2009; 180:365-370. [PubMed: 19483111]

13. Yoon YS, Lee HJ, Yoon HI, et al. Impact of fluoroquinolones on the diagnosis of pulmonary tuberculosis initially treated as bacterial pneumonia. Int J Tuberc Lung Dis. 2005; 9:1215-1219. [PubMed: 16333927]

14. Wang JY, Hsueh PR, Jan IS, et al. Empirical treatment with a fluoroquinolone delays the treatment for tuberculosis and is associated with a poor prognosis in endemic areas. Thorax. 2006; 61:903908. [PubMed: 16809417] 
15. Jeon CY, Calver AD, Victor TC, Warren RM, Shin SS, Murray MB. Use of fluoroquinolone antibiotics leads to tuberculosis treatment delay in a South African gold mining community. Int J Tuberc Lung Dis. 2011; 15:77-83. [PubMed: 21276301]

16. Dooley KE, Golub J, Goes FS, Merz WG, Sterling TR. Empiric treatment of community-acquired pneumonia with fluoroquinolones, and delays in the treatment of tuberculosis. Clin Infect Dis. 2002; 34:1607-1612. [PubMed: 12032896]

17. Golub JE, Bur S, Cronin WA, et al. Impact of empiric antibiotics and chest radiograph on delays in the diagnosis of tuberculosis. Int J Tuberc Lung Dis. 2005; 9:392-397. [PubMed: 15832463]

18. Català L, Orcau A, García de Olalla P, Millet J-P, Rodríguez-Mondragón A, Caylà JA. Survival of a large cohort of HIV-infected tuberculosis patients in the era of highly active antiretroviral treatment. Int J Tuberc Lung Dis. 2011; 15:263-269. [PubMed: 21219692]

19. Oursler KK, Moore RD, Bishai WR, Harrington SM, Pope DS, Chaisson RE. Survival of patients with pulmonary tuberculosis: clinical and molecular epidemiologic factors. Clin Infect Dis. 2002; 34:752-759. [PubMed: 11850859]

20. US Census Bureau. 2009 Regional tuberculosis case and rate table with population estimates. Washington, DC, USA: US Census Bureau; 2009. http://health.state.tn.us/Downloads/ TB_2009_bycounty.pdf [Accessed May 2012]

21. Wang M, FitzGerald JM, Richardson K, et al. Is the delay in diagnosis of pulmonary tuberculosis related to exposure to fluoroquinolones or any antibiotic? Int J Tuberc Lung Dis. 2011; 15:10621068. [PubMed: 21740669]

22. Chen TC, Lu PL, Lin CY, Lin WR, Chen YH. Fluoroquinolones are associated with delayed treatment and resistance in tuberculosis: a systematic review and meta-analysis. Int J Infect Dis. 2011; 15:e211-e216. [PubMed: 21195001] 


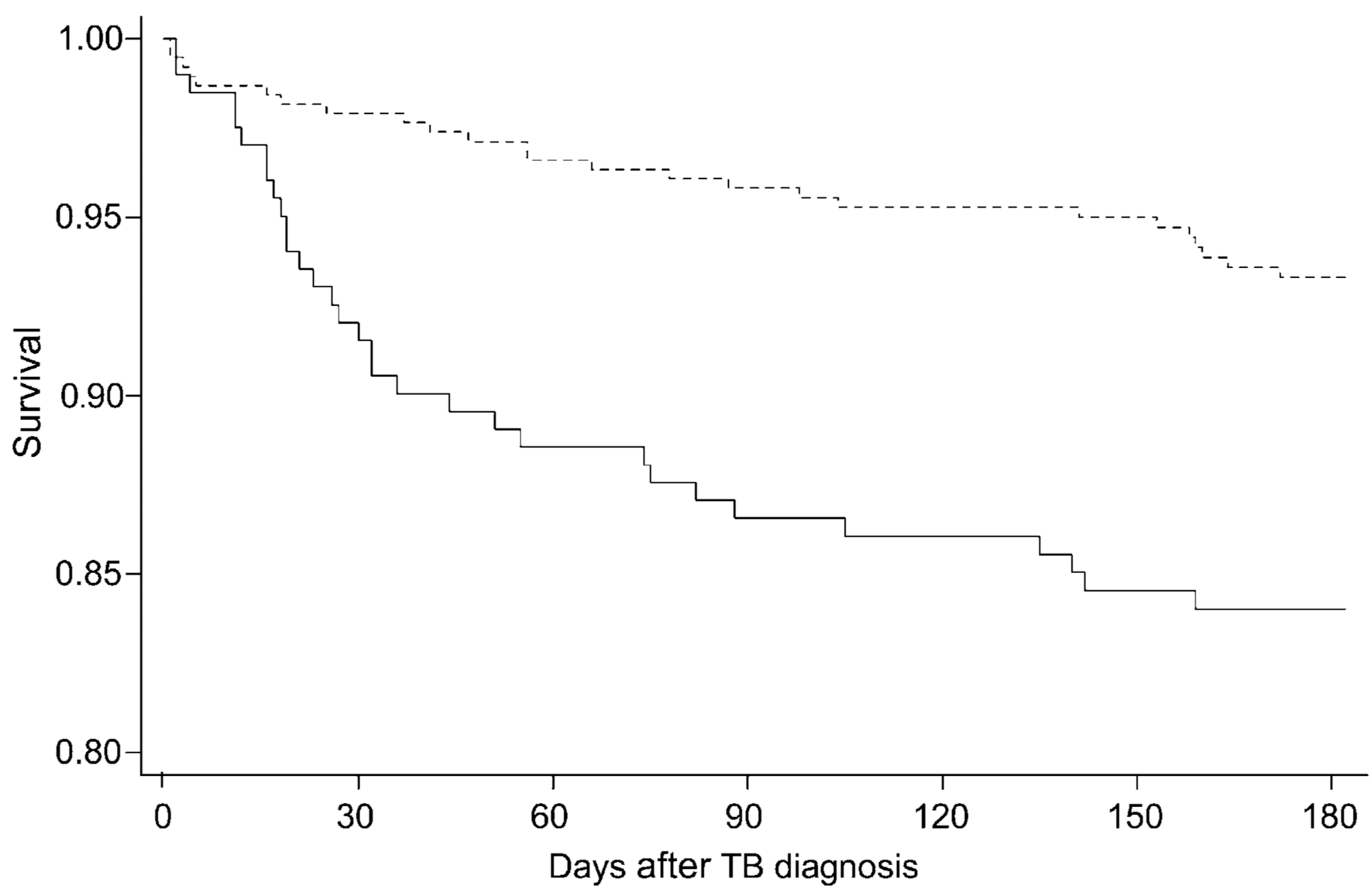

\section{---- No FQ before TB diagnosis — Received any FQ before TB diagnosis}

Figure.

Kaplan-Meier survival estimates in the first 182 days after TB diagnosis comparing those who were exposed to FQ during the 6 months preceding TB diagnosis with those who were not. $\mathrm{TB}=$ tuberculosis; $\mathrm{FQ}=$ fluoroquinolones. 
Table 1

Demographic, clinical and drug resistance characteristics

\begin{tabular}{lccc}
\hline & $\begin{array}{c}\text { Any } \\
\text { FQ exposure } \\
(\boldsymbol{n}=\mathbf{2 1 4}) \\
\text { Characteristic }\end{array}$ & $\begin{array}{c}\text { NQ } \\
\text { FQ exposure } \\
(\boldsymbol{n}=\mathbf{3 9 5}) \\
\boldsymbol{n}(\boldsymbol{\%})\end{array}$ & P value \\
\hline Age, years, median [IQR] & $53[40-73]$ & $43[29-59]$ & $<0.001$ \\
Male & $134(63)$ & $256(65)$ & 0.60 \\
Black race & $79(37)$ & $159(40)$ & 0.42 \\
Hispanic ethnicity & $24(11)$ & $69(18)$ & 0.04 \\
US-born & $173(81)$ & $261(66)$ & $<0.001$ \\
HIV-positive & $25 / 201(12)$ & $34 / 385(9)$ & 0.17 \\
Drug resistance & & & \\
FQs & $8 / 176(5)$ & $1 / 265(0)$ & 0.002 \\
Isoniazid & $7 / 183(4)$ & $6 / 271(2)$ & 0.31 \\
Rifampin & $3 / 184(2)$ & $0 / 271(0)$ & 0.04 \\
Pyrazinamide & $4 / 180(2)$ & $11 / 268(4)$ & 0.28 \\
Ethambutol & $2 / 184(1)$ & $0 / 271(0)$ & 0.09 \\
\hline
\end{tabular}

$\mathrm{FQ}=$ fluoroquinolone; $\mathrm{IQR}=$ interquartile range; $\mathrm{HIV}=$ human immunodeficiency virus. 
Table 2

\section{FQ exposure characteristics}

\begin{tabular}{lc}
\hline Variable & $\begin{array}{c}\text { Patients } \\
(\boldsymbol{n}=\mathbf{6 0 9}) \\
\boldsymbol{n}(\boldsymbol{\%})\end{array}$ \\
\hline Any FQ exposure & $214(35)$ \\
Duration of FQ exposure prior to TB diagnosis, days, median [IQR] & $7[3-14]$ \\
$>10$ & $90(15)$ \\
$1-10$ & $124(20)$ \\
Timing of exposure, days prior to TB diagnosis, median [IQR $]^{*}$ & $8.5[0-35]$ \\
Receipt of FQ $>60$ days prior to TB diagnosis & $31(5)$ \\
Receipt of FQ 50 days prior to TB diagnosis & $151(25)$ \\
Specific FQ ${ }^{\dagger}$ & \\
Moxifloxacin & $73(12)$ \\
Levofloxacin & $127(21)$ \\
Ciprofloxacin & $68(11)$ \\
Ofloxacin & 0 \\
Gatifloxacin & 0 \\
\hline
\end{tabular}

*nly 182 cases with known timing of exposure included.

${ }^{\dagger}$ Number of cases who received at least one prescription of each FQ; some persons received more than one type of FQ.

$\mathrm{FQ}=$ fluoroquinolone; $\mathrm{TB}$ = tuberculosis; $\mathrm{IQR}=$ interquartile range. 


\section{Table 3}

Factors associated with death at the time of diagnosis or during tuberculosis treatment ${ }^{*}$

\begin{tabular}{llclc}
\hline Risk factor & $\begin{array}{l}\text { Unadjusted } \\
\text { OR }(95 \% \mathrm{CI})\end{array}$ & $P$ value & $\begin{array}{l}\text { Adjusted } \\
\text { OR }(95 \% \mathrm{CI})^{\dagger}\end{array}$ & $P$ value \\
\hline Age & $1.05(1.03-1.06)$ & $<0.001$ & $1.05(1.04-1.07)$ & $<0.001$ \\
Male sex & $1.39(0.81-2.38)$ & 0.24 & & \\
Black race & $1.24(0.75-2.04)$ & 0.40 & & \\
HIV-positive & $4.41(2.29-8.50)^{\dagger}$ & $<0.001^{+}$ & $8.08(3.83-17.06)$ & $<0.001$ \\
Fluoroquinolone exposure & $2.70(1.63-4.46)$ & $<0.001$ & $1.82(1.05-3.15)$ & 0.03 \\
US-born & $7.80(2.80-21.75)$ & $<0.001$ & $3.03(1.03-9.09)$ & 0.04 \\
Any first-line drug resistance $\$$ & $0.62(0.14-2.68)$ & 0.52 & & \\
\hline
\end{tabular}

* The unadjusted model included MDR-TB. However, no MDR-TB patients were dead at diagnosis or died during anti-tuberculosis treatment.

${ }^{\dagger}$ Multivariable regression results applied multiple imputation techniques for those with unknown HIV status.

${ }^{\ddagger}$ Calculated using only those with known HIV status $(n=586)$.

$\S$ Drug resistance to any of isoniazid, rifampin, pyrazinamide, ethambutol.

$\mathrm{OR}=$ odds ratio CI = confidence interval HIV = human immunodeficiency virus; $\mathrm{MDR}-\mathrm{TB}=$ multidrug-resistant tuberculosis . 
Table 4

Association of FQ exposure with clinical, radiological and bacteriological presentation

\begin{tabular}{lccc}
\hline & $\begin{array}{c}\text { Any } \\
\text { FQ exposure } \\
(\boldsymbol{n = 2 1 4 )} \\
\boldsymbol{n}(\boldsymbol{\%})\end{array}$ & $\begin{array}{c}\text { No } \\
\text { FQ exposure } \\
(\boldsymbol{n}=\mathbf{3 9 5}) \\
\boldsymbol{n}(\mathbf{\%})\end{array}$ & P value \\
Outcome & $40(19)$ & $31(8)$ & $<0.001$ \\
\hline Death, all & $6(3)$ & $4(1)$ & 0.10 \\
At the time of TB diagnosis & $34(16)$ & $27(7)$ & $<0.001$ \\
While being treated for TB & & & 0.19 \\
Site of disease & $170(79)$ & $294(74)$ & \\
Pulmonary only & $36(17)$ & $73(18)$ & \\
Extra-pulmonary only & $8(4)$ & $28(7)$ & \\
Both pulmonary and extra-pulmonary & $60(28)$ & $90(23)$ & 0.15 \\
Cavitary disease & & & $<0.001$ \\
Culture status & $186(87)$ & $274(69)$ & \\
Culture-positive TB & $28(13)$ & $121(31)$ & \\
Culture-negative TB & & & $<0.001$ \\
Smear status & $107(50)$ & $134(34)$ & \\
Smear-positive TB & $107(50)$ & $261(66)$ & \\
Smear-negative TB & & & \\
\hline
\end{tabular}

$\mathrm{FQ}=$ fluoroquinolone; $\mathrm{TB}=$ tuberculosis. 